\title{
On the maximal exponent of the prime power divisor of integers
}

\author{
Imre Kátai \\ Faculty of Informatics \\ Eötvös Loránd University \\ Budapest, Hungary \\ email: katai@inf.elte.hu
}

\author{
Bui Minh Phong \\ Faculty of Informatics \\ Eötvös Loránd University \\ Budapest, Hungary \\ email: bui@inf.elte.hu
}

Abstract. The largest exponent of the prime powers function is investigated on the set of numbers of form one plus squares of primes.

\section{Introduction}

1.1. Notation. Let, as usual, $\mathcal{P}, \mathbb{N}$ be the set of primes, positive integers, respectively. For a prime divisor $p$ of $n$ let $v_{p}(n)$ be defined by $p^{v_{p}(n)} \| n$. Then $n=\prod_{p \mid n} p^{v_{p}(n)}$. Let

$$
H(n)=\max _{p \mid n} v_{p}(n) \quad \text { and } \quad h(n)=\min _{p \mid n} v_{p}(n) .
$$

We denote by $\pi(x)$ the number of primes $p \leq x$ and by $\pi(x, k, \ell)$ the number of primes $p \leq x, p \equiv \ell(\bmod k)$.

1.2. Preliminaries. A. Niven proved in [7] that

$$
\sum_{n \leq x} h(n)=x+\frac{\zeta(3 / 2)}{\zeta(3)} \sqrt{x}+o(\sqrt{x}) \quad(x \rightarrow \infty)
$$

2010 Mathematics Subject Classification: 11A25, 11N25, 11N64

Key words and phrases: the prime powers function, the set of one plus squares of primes 
and that

$$
\frac{1}{x} \sum_{n \leq x} H(n) \rightarrow B \quad(x \rightarrow \infty), \text { where } \quad B=1+\sum_{k=2}^{\infty}\left(1-\frac{1}{\zeta(k)}\right) .
$$

W. Schwarz and J. Spilker showed in [8] that

$$
\begin{aligned}
& \sum_{n \leq x} H(n)=\mathcal{M}(H) x+O\left(x^{3 / 4} \exp (-\gamma \sqrt{\log x})\right) \quad(x \rightarrow \infty), \\
& \sum_{n \leq x} \frac{1}{H(n)}=\mathcal{M}\left(\frac{1}{H}\right) x+O\left(x^{3 / 4} \exp (-\gamma \sqrt{\log x)} \quad(x \rightarrow \infty),\right.
\end{aligned}
$$

where $\gamma>0$ is a suitable constant, $\mathcal{M}(\mathrm{H})=\mathrm{B}, \mathcal{M}\left(\frac{1}{\mathrm{H}}\right)$ are suitable positive numbers.

D. Suryanayana and Sita Ramachandra Rao [9] proved that the error term in (3) and (4) can be improved to

$$
\mathrm{O}\left(\sqrt{x} \exp \left(-\gamma(\log x)^{3 / 5}(\log \log x)^{-1 / 5}\right)\right) .
$$

They proved furthermore that

$$
\begin{aligned}
& \sum_{n \leq x} h(n)=c_{1} x+c_{2} x^{1 / 2}+c_{3} x^{1 / 3}+c_{4} x^{1 / 4}+c_{5} x^{1 / 5}+O\left(x^{1 / 6}\right) \\
& \sum_{n \leq x} \frac{1}{h(n)}=d_{1} x+d_{2} x^{1 / 2}+d_{3} x^{1 / 3}+d_{4} x^{1 / 4}+d_{5} x^{1 / 5}+O\left(x^{1 / 6}\right)
\end{aligned}
$$

Gu Tongxing and Cao Huizhong announced in [4] that they can improve the error term in (3) to

$$
\mathrm{O}\left(\sqrt{x} \exp \left(-\mathrm{c}(\log x)^{3 / 5}(\log \log x)^{-1 / 5}\right)\right) .
$$

I. Kátai and M. V. Subbarao [5] investigated the asymptotic of

$$
A_{x}(r):=\left\llcorner\{n \in[x, x+Y] \mid H(n)=r\}, \quad Y=x^{\frac{1}{2 r+1}} \log x,\right.
$$

and

$$
\mathrm{B}_{\chi}(\mathrm{r}):=\left\lfloor\{\mathrm{p} \in \mathcal{P}, \mathrm{p} \in[x, x+\mathrm{Y}] \mid \mathrm{H}(\mathrm{p}+1)=\mathrm{r}\}, \quad \mathrm{Y}=x^{\frac{7}{12}+\epsilon}\right.
$$

for fixed $r \geq 1$. 
Namely, they proved that

$$
A_{x}(r)=Y(\eta(r+1)-\eta(r))+O\left(\frac{Y}{\log x}\right), \eta(s)=\frac{1}{\zeta(s)}-1 \quad(s=1,2, \cdots)
$$

and

$$
B_{x}(r)=e(r) \frac{Y}{\log x}+O\left(\frac{Y}{(\log x)^{2}}\right)
$$

where

$$
e(1)=\prod_{p \in \mathcal{P}}\left(1-\frac{1}{p(p-1)}\right)
$$

and for $r \geq 2$

$$
e(r)=\prod_{p \in \mathcal{P}}\left(1-\frac{1}{(p-1) p^{r}}\right)-\prod_{p \in \mathcal{P}}\left(1-\frac{1}{(p-1) p^{r-1}}\right) .
$$

In [6] we can read some results on (5) assuming the Riemann conjecture.

Our main interest now is to give the asymptotic of the number of those $n \leq x, n \in \mathcal{B}$, for which $H(n)=r$ uniformly as $1 \leq r \leq k(x)$, where $k(x)$ is as large as it is possible. We shall investigate it when $\mathcal{B}=$ set of shifted primes.

\subsection{Auxiliary results.}

Lemma 1 (Brun-Titchmarsh inequality). We have

$$
\pi(x, k, \ell)<C \frac{x}{\varphi(k) \log \frac{x}{k}} .
$$

Lemma 2 (Siegel-Walfisz theorem). We have

$$
\pi(x, k, \ell)=\frac{\operatorname{lix}}{\varphi(k)}\left(1+\mathrm{O}\left(e^{-\mathrm{c} \sqrt{\log x}}\right)\right)
$$

uniformly as $(\mathrm{k}, \ell)=1, \mathrm{k} \leq(\log \mathrm{x})^{\mathrm{A}}$. Here $\mathrm{A}$ is arbitrary, $\mathrm{c}>0$ is a fixed constant.

Lemma 3 ([1]) Let $\mathrm{q}$ be an odd prime, $\mathrm{D}=\mathrm{q}^{\mathrm{n}}(\mathrm{n}=1,2, \cdots), \epsilon>0$ be an arbitrary small, and $\mathrm{M}$ be an arbitrary large positive number. Then the asymptotic law

$$
\pi(x, D, \ell)=\frac{\operatorname{lix}}{\varphi(D)}\left(1+\mathrm{O}\left((\log x)^{-M}\right)\right)
$$

holds for $\mathrm{D} \leq \mathrm{x}^{3 / 8-\epsilon},(\ell, \mathrm{D})=1$. 
Lemma 4 ([2]) Let $\mathrm{a}$ be an integer, $\mathrm{a} \geq 2$. If $\mathrm{A}>0$, then there is $a \mathrm{~B}>0$ for which

$\sum_{\substack{d \leq \frac{x^{1 / 2}}{q}(\log x)^{-B} \\(d, q)=1}} \max _{(r, q d)=1} \max _{y \leq x}\left|\pi(y, q d, r)-\frac{\operatorname{lix}}{\varphi(q d)}\right| \ll \frac{x}{\varphi(q)(\log x)^{A}}, \operatorname{lix}=\int_{2}^{x} \frac{d u}{\log u}$ uniformly for moduli $\mathrm{q} \leq x^{1 / 3} \exp \left(-(\log \log x)^{3}\right)$ that are powers of $\mathrm{a}$.

While the implicit constant in $\ll$ may depend upon $\mathrm{a}, \mathrm{B}$ is a function of $\mathrm{A}$ alone. $\mathrm{B}=\mathrm{A}+6$ is permissible.

We shall use a special consequence of this assertion:

Corollary. Let $\mathrm{a}$ be an integer, $\mathrm{a} \geq 2, \mathrm{D}=\mathrm{a}^{\mathrm{n}} \quad(\mathrm{n}=1,2, \cdots), \mathrm{D} \leq$ $x^{1 / 3} \exp \left(-(\log \log x)^{3}\right)$. Let $\mathrm{A}>0$ be an arbitrary constant. Then

$$
\pi(x, D, \ell)=\frac{\operatorname{lix}}{\varphi(D)}\left(1+O\left(\frac{1}{(\log x)^{A}}\right)\right), \quad(\ell, D)=1
$$

Lemma 5 ([3]) Let $\mathrm{q}=\mathrm{p}^{\mathrm{r}}, \mathrm{p}$ an odd prime, $\mathrm{q} \mathrm{x}^{\frac{3}{5}+\epsilon} \leq \mathrm{h} \leq \mathrm{x}$. Then

$$
\pi(x+h, q, \ell)-\pi(x, q, \ell)=\left(1+o_{x}(1)\right) \frac{h}{\varphi(q) \log x}
$$

as $x \rightarrow \infty,(\ell, q)=1$.

\section{Formulation of the theorems}

Let $(0<) \mathrm{U}, \mathrm{V}$ be coprime integers, and let $\mathrm{Q}$ be the smallest prime for which

$$
\mathrm{U}(1+2 \mathrm{~m})+\mathrm{V} \equiv 0 \quad(\bmod \mathrm{Q})
$$

has a solution, that is

$$
\mathrm{Q}=\left\{\begin{array}{l}
2 \text { if } 2 \mid \mathrm{U}+\mathrm{V} \\
\text { smallest prime for which }(\mathrm{Q} 2 \mathrm{U})=1, \text { if } 2 \nmid \mathrm{U}+\mathrm{V} .
\end{array}\right.
$$

Let

$$
M_{U, V}(x \mid k)=\llcorner\{p \leq x \mid H(U p+V)=k\} .
$$


Theorem 1 Assume that $\mathrm{r}(\mathrm{x}) \rightarrow \infty$ arbitrarily slowly. Then, in the interval $r(x)<k<\left(\frac{1}{3}-\epsilon\right) \frac{\log x}{\log Q}$, we have

$$
\mathrm{M}_{\mathrm{u}, \mathrm{V}}(\mathrm{x} \mid \mathrm{k})=\frac{\mathrm{lix}}{\varphi\left(\mathrm{Q}^{\mathrm{k}}\right)}\left(1-\frac{1}{\mathrm{Q}}\right) \cdot\left(1+\mathrm{o}_{\chi}(1)\right) .
$$

Let $P(n)=n^{2}+1$. Then $4 \nmid P(n), 3 \nmid P(n), 5|P(2), 5| P(3)$. For every $k$ there exists $1 \leq \ell_{k}<\frac{5^{k}}{2}$, such that $P\left(\ell_{k}\right) \equiv 0\left(\bmod 5^{k}\right)$. The congruence $\mathrm{P}(\mathrm{n}) \equiv 0\left(\bmod 5^{\mathrm{k}}\right)$ has exactly two solutions: $\ell_{k}$ and $5^{k}-\ell_{k}$. It obvious that $\left(\ell_{k}, 5\right)=1$.

Let

$$
E(x \mid k)=a\left\{p \leq x \mid H\left(p^{2}+1\right)=k\right\} .
$$

Theorem 2 Assume that $\mathrm{r}(\mathrm{x}) \rightarrow \infty$ arbitrarily slowly. Then, in the interval $r(x)<k<\left(\frac{1}{3}-\epsilon\right) \frac{\log x}{\log 5}$, we have

$$
E(x \mid k)=\frac{2}{5^{k}} \operatorname{lix}\left(1+o_{x}(1)\right)
$$

\section{Proof of Theorem 1.}

It is obvious that

$$
\mathrm{M}_{\mathrm{U}, \mathrm{V}}(x \mid \mathrm{k}) \leq \sum_{\mathrm{q}}^{*}\left[\mathrm{Q}\left(x, \mathrm{q}^{\mathrm{k}}, \mathrm{r}_{\mathrm{q}, \mathrm{k}}\right)-\mathrm{Q}\left(x, \mathrm{q}^{\mathrm{k}+1}, \mathrm{r}_{\mathrm{q}, \mathrm{k}+1}\right)\right]
$$

where $\mathrm{q}$ runs over all those primes for which $\mathrm{U}(1+2 \mathrm{~m})+\mathrm{V} \equiv 0(\bmod \mathrm{q})$ has a solution, $\mathrm{r}_{\mathrm{q}, \mathrm{k}} \equiv \mathrm{VU}^{-1}\left(\bmod \mathrm{q}^{\mathrm{k}}\right), \mathrm{r}_{\mathrm{q}, \mathrm{k}+1} \equiv \mathrm{Vu}^{-1}\left(\bmod \mathrm{q}^{\mathrm{k}+1}\right)$.

By using Lemma 3 and Lemma 1 we obtain that

$$
\begin{aligned}
\mathrm{M}_{\mathrm{U}, \mathrm{V}}(\mathrm{x} \mid \mathrm{k}) & \leq \frac{\operatorname{lix}}{\varphi\left(\mathrm{Q}^{\mathrm{k}}\right)}\left(1-\frac{1}{\mathrm{Q}}\right) \cdot\left(1+\mathrm{O}\left(\frac{1}{(\log x)^{\mathrm{M}}}\right)\right)+ \\
& +\mathrm{C} \sum_{\substack{\mathrm{q}>\mathrm{Q} \\
\mathrm{q} \in \mathcal{P}}} \frac{\mathrm{lix}}{\varphi\left(\mathrm{q}^{\mathrm{k}}\right)}+\mathrm{C} \sum_{\substack{\mathrm{Q}<\mathrm{q} \\
\mathrm{q}^{\mathrm{k}} \geq \sqrt{\mathrm{x}}}} \frac{x}{\mathrm{q}^{\mathrm{k}}} .
\end{aligned}
$$

It is clear that

$$
\sum_{\substack{q>Q \\ q \in \mathcal{P}}} \frac{1}{\varphi\left(q^{k}\right)}=\frac{o_{x}(1)}{\varphi\left(Q^{k}\right)}
$$


and that

$$
\sum_{\substack{k \\ q \in \sqrt{x}}} \frac{1}{\varphi\left(q^{k}\right)}=O\left(\frac{1}{x^{1 / 4}}\right)
$$

thus

$$
\mathrm{M}_{\mathrm{U}, \mathrm{v}}(x \mid \mathrm{k}) \leq\left(1+\mathrm{o}_{x}(1)\right) \frac{\mathrm{lix}}{\mathrm{Q}^{\mathrm{k}}} .
$$

On the other hand

$$
M_{\mathrm{U}, \mathrm{V}}(x \mid \mathrm{k}) \geq\left[\mathrm{Q}\left(x, \mathrm{Q}^{\mathrm{k}}, \mathrm{r}_{\mathrm{Q}, \mathrm{k}}\right)-\mathrm{Q}\left(x, \mathrm{Q}^{\mathrm{k}+1}, \mathrm{r}_{\mathrm{Q}, \mathrm{k}+1}\right)\right]-\sum_{\substack{\mathrm{q}>\mathrm{Q} \\ \mathrm{q} \in \mathcal{P}}} \mathrm{Q}\left(x, \mathrm{Q}^{\mathrm{k}} \mathrm{q}^{\mathrm{k}}, \mathrm{r}_{\mathrm{Qq}, \mathrm{k}}\right) .
$$

The sum on right hand side is less than

$$
\mathrm{C} \frac{\operatorname{li} x}{\mathrm{Q}^{\mathrm{k}}} \sum_{(\mathrm{Q}<) \mathrm{q}} \frac{1}{\mathrm{q}^{\mathrm{k}}}+\mathrm{O}\left(\mathrm{x}^{3 / 4}\right) \leq \mathrm{o}_{x}(1) \frac{\mathrm{lix}}{\mathrm{Q}^{\mathrm{k}}} .
$$

From Lemma 3 our theorem follows.

\section{Proof of Theorem 2}

We have

$$
E(x \mid k)=S+O(T),
$$

where

$$
S=\left\llcorner\left\{p \leq x: \quad 5^{k} \| p^{2}+1\right\}\right.
$$

and

$$
\mathrm{T}=\sum_{\substack{\mathrm{q} \in \mathcal{P} \\ \mathrm{q}>5}} \downarrow\left\{\mathrm{p} \leq x: \quad \mathrm{q}^{\mathrm{k}} \| \mathrm{p}^{2}+1\right\} .
$$

Thus, by using Lemma 1 and $k \geq \gamma(x)$,

$$
\mathrm{T} \leq \sum_{\substack{\mathrm{q} \in \mathcal{P} \\ \mathrm{q}>5}} \frac{2 \text { Clix }}{\varphi\left(\mathrm{q}^{\mathrm{k}}\right)}+\sum_{\substack{\mathrm{q}^{\mathrm{k}>x} \\ \mathrm{q} \in \mathcal{P}}} \frac{x}{\mathrm{q}^{\mathrm{k}}}=\mathrm{o}_{x}(1) \frac{\operatorname{lix}}{5^{\mathrm{k}}}
$$

Hence we obtain that

$$
E(x \mid k) \leq \frac{2}{5^{k}} \operatorname{lix}\left(1+o_{x}(1)\right) .
$$


On the other hand

$$
E(x \mid k) \geq S-\sum_{\substack{q \in \mathcal{P} \\ q>5}} \boxminus\left\{p \leq x: 5^{k} \cdot q^{k} \| p^{2}+1\right\} .
$$

By using Lemma 1, the sum on the right can be overestimated by

$$
\frac{\operatorname{Cli} x}{5^{k}} \sum_{q>5} \frac{1}{\varphi\left(q^{k}\right)}+\frac{x}{5^{k}} \sum_{q^{k}>\sqrt{x}} \frac{1}{q^{k}},
$$

which is clearly $o_{x}(1) S$.

This completes the proof of Theorem 2 .

\section{$5 \quad$ Further remarks}

By using Lemma 5 we can prove short interval version of Theorem 1 and 2 .

Theorem 3 Let $5^{k} x^{3 / 5+\epsilon} \leq h \leq x, k \geq g(x)$. Then

$$
E(x+h \mid k)-E(x)=\frac{h}{5^{k}} \frac{1}{\log x}\left(1+o_{x}(1)\right) .
$$

Theorem 4 Let Let $\mathrm{U}, \mathrm{V}$ be coprime integers, $\mathrm{U}>0, \mathrm{U}+\mathrm{V}=$ odd, $\mathrm{Q}$ be the smallest prime which is not a divisor of $2 \mathrm{U}$. Let $\mathrm{k} \geq \mathrm{g}(\mathrm{x}), \mathrm{Q}^{\mathrm{k}} \mathrm{x}^{3 / 5+\epsilon} \leq \mathrm{h} \leq \mathrm{x}$. Then

$$
\mathrm{M}_{\mathrm{U}, \mathrm{V}}(\mathrm{x}+\mathrm{h} \mid \mathrm{k})-\mathrm{M}_{\mathrm{U}, \mathrm{V}}(\mathrm{x})=\left(1+\mathrm{o}_{\mathrm{x}}(1)\right) \frac{\mathrm{h}}{\mathrm{Q}^{\mathrm{k}}} \frac{1}{\log \mathrm{x}} \text { as } \mathrm{x} \rightarrow \infty
$$

\section{Acknowledgements}

This work was completed with the support of the Hungarian and Vietnamese TET (grant agreement no. TET 10-1-2011-0645).

\section{References}

[1] M. B. Barban, Yu. V. Linnik, N. G. Tshudakov, On prime numbers in an arithmetic progression with a prime-power difference, Acta Arithmetica, 9 (1964), 375-390. 
[2] P. D. T. A. Elliott, Primes in progressions to moduli with a large power factor, Ramanujan J., 13 (2007), 241-251.

[3] P. X. Gallagher, Primes to progressions to prime-power modulus, Inventiones Math, 16 (1972), 191-201.

[4] Gu Tongxing and Cao Huizhong, On sums of exponents of factoring integers, Journal of Mathematical Research and Exposition, 13 (1993), 166.

[5] I. Kátai and M. V. Subbarao, On the maximal and minimal exponent of the prime power divisors of integers, Publ. Math. Debrecen, 68 (2006), $477-488$.

[6] Kaneeika Sinka, Average orders of certain arithmetical functions, $J$. Ramanujan Math. Soc., 21 (3) (2006), 267-277; corrigendum ibid. 24 (2) (2009), 211.

[7] I. Niven, Averages of exponents in factoring integers, Proc. Amer. Math. Soc., 22 (1969), 356-360.

[8] W. Schwarz, J. Spilker, A remark on some special arithmetical functions, New Trends in Prob. and Stat., 4 (1996), 221-245.

[9] D. Suryanayana and Sita Ramachandra Rao, On the maximum and minimum exponents in factoring integers, Archiv Math, 28 (1977),261269. 